\title{
The subjective assessment of the effect and satisfaction with dermocosmetics use by patients with skin disturbances
}

\author{
Magdalena Olszanecka-Glinianowicz
}

Health Promotion and Obesity Management Unit, Department of Pathophysiology, Medical Faculty, Medical University of Silesia, Katowice, Poland

Head of the Unit: Prof. Magdalena Olszanecka-Glinianowicz MD, PhD

Postep Derm Alergol 2015; XXXII (3): 195-203

DOI: 10.5114/pdia.2015.51751

\begin{abstract}
Introduction: Atopic dermatitis and different constellations and the severity of symptoms that not meet the criteria for this diagnosis are a common skin disturbances. An important component of the treatment of these diseases and the proper care of sensitive and dry skin is local dermocosmetics use.

Aim: To assess the frequency of skin disturbances and the effect and satisfaction with Atoperal products use as well as the source of information about these products.

Material and methods: A questionnaire survey about the type of skin disturbances, the subjective assessment of the effect and satisfaction with Atoperal products use and source of information about these products was performed by 787 general practitioners, internists, pediatricians, dermatologists, allergists and pulmonologists and 252 pharmacists in a group of 51085 subjects with skin disturbances.

Results: In the group interviewed by doctors, the most common skin problem was atopic dermatitis (52.5\%) and in the group interviewed by pharmacists, pruritus (70.0\%). In both groups, respondents after Atoperal products use most frequently reported improving of the skin hydration and greasiness of the skin and reduction of itching. In both groups, over $90.0 \%$ of respondents were satisfied or very satisfied with Atoperal products use. In the group surveyed by doctors, $75.5 \%$ of respondents obtained information about these products from doctors and $17.4 \%$ from pharmacists, while in the group surveyed by pharmacists, $48.9 \%$ from pharmacists and $36.1 \%$ from doctors. Conclusions: Atopic dermatitis was most frequently diagnosed in a group surveyed by doctors. The main skin disturbance that occurred in a group surveyed by pharmacists was skin pruritus. The main effect of Atoperal products use, independent of the place of the survey, included improving of skin hydration and greasiness of the skin and reduction of itching. In a study population, there was a high level of satisfaction from the use of Atoperal products. Doctors and pharmacists were the main sources of information about Atoperal products depending on the place of the survey.
\end{abstract}

Key words: atopic dermatitis, other skin disturbances, dermocosmetics.

\section{Introduction}

The prevalence of atopic dermatitis in children from industrialized countries is estimated to be $5-20 \%$. It has also been shown that the incidence of atopic dermatitis in numerous countries has increased from 3 to 5 times. However, in adult populations, there is a decrease due to the self-limiting nature of this disease (the symptoms of dermatitis after puberty persist in $5-10 \%$ of patients only). In addition, severity of symptoms decreased in a significant percentage of patients [1].
Atopic dermatitis is a chronic disease, genetically determined and its pathogenesis has not been fully explained. For the diagnosis of atopic dermatitis, at least 3 of the 4 main criteria are required to be met including persistent pruritus, typical changes in morphology and location, chronicity and recurrence, and a personal and/ or family history of atopic diseases and three additional ones including dry skin, ichthyosis, pilaris keratosis, cheilitis, inflamed nipples, recurrent conjunctivitis, increased levels of IgE, food and/or wool in tolerance, the impact

Address for correspondence: Prof. Magdalena Olszanecka-Glinianowicz MD, PhD, Health Promotion and Obesity Management Unit, Department of Pathophysiology, 18 Medyków St, 40-752 Katowice, Poland, phone/fax: +48 322526 091, e-mail: magols@esculap.pl Received: 14.05.2015, accepted: 25.05.2015. 
of stress and environmental factors on exacerbations, recurrent skin infections and other features [2]. Some patients may not meet the criteria for the diagnosis of atopic dermatitis, but have different constellations and the severity of the symptoms listed above [2].

An important component of the treatment of these diseases and the proper care of sensitive and dry skin is local dermocosmetics use. Dermocosmetics provide proper hydration and restoration of the damaged lipid barrier, mainly by providing deficient lipid components, as well as the restoration of correct hydration of the stratum corneum. However, it should be noted that the therapeutic effect is provided by hypoallergenic cosmetics which contain emollients in the form of soaps, gels, shampoos, lotions, and creams. These dermocosmetics have also anti-inflammatory properties and their use reduce the severity of itching and make the skin more flexible and form a protective film [3]. One type of dermocosmetics available on the Polish market is Atoperal and Atoperal Baby products. Numerous factors may influence the first choice of dermocosmetics, for example advertising, the recommendation by a friend or pharmacist or doctor and the cost of the brand manufacturer, gender, age, place of residence, education level and type of skin disturbances. However, the continued use of dermocosmetics may largely depend on the satisfaction related to ameliorating the symptoms of the disease. The factors influencing the choice and satisfaction with the use of Atoperal and Atoperal Baby products are unknown.

\section{Aim}

Therefore, the aims of the study were: the assessment of the frequency of skin disturbances in relation to socio-demographic characteristics of respondents depending on the place of completing the survey. Determination of the subjective assessment of the effect of the Atoperal and Atoperal Baby products use on skin symptoms and satisfaction with their use depending on the place of completing the survey. The assessment of the sources of information about the Atoperal and Atoperal Baby products depending on the place of completing the survey.

\section{Material and methods}

In this observational survey, 51085 respondents were interviewed nation wide by 787 general practitioners, internists, pediatricians, dermatologists, allergists and pulmonologists as well as 252 pharmacists in 2013-2014. Polish doctors and pharmacists participating in the study were recruited by medical representatives, and each of them conducted questionnaire interviews with a group of 51085 consecutive patientsor guardians of children referred to the clinic or pharmacy for skin symptoms and using dermocosmetics.
Characteristics of the surveyed population are summarized in Table 1.

The questionnaire consisted of several dichotomous and multiple choice questions.

The questionnaire included demographic data (gender, age range, place of residence, education levels), type of skin disturbances (dry skin, pruritus, atopic dermatitis, tendency to recurrent infections of the skin, other). In addition, the current or past use of Atoperal or Atoperal Baby products and their type have been reported. Respondents have also assessed the effect of Atoperal or Atoperal Baby products on the appearance and condition of the skin (skin hydration improvement/improvement of greasiness of the skin/skin elasticity increase/decrease of itching/acceleration of healing) and the degree of satisfaction with these products use. In addition, the source of information about Atoperal and Atoperal Baby products (doctor/pharmacist/friends/promotional handouts/Internet/newspaper/other) were recorded.

\section{Statistical analysis}

Statistical analysis was performed using the Statistica 10.0 PL (Cracow, Poland) software package.

The socio-demographic data were analyzed depending on the place of completing the survey. The subjective assessment of the effect of Atoperal and Atoperal Baby products use on skin symptoms and satisfaction with their use depending on the place of completing the survey were also assessed.

Values of variables were presented as percentages and mean values with standard deviations (SD). Separate groups were compared using the $\chi^{2}$ test and $\chi^{2}$ test for trend. A $p<0.05$ was considered as statistically significant.

\section{Results}

Skin symptoms occurring in the study group in relation to the place of the survey

In a group interviewed by doctors, the most common skin problem was atopic dermatitis (52.5\%) and in the group interviewed by pharmacists it was pruritus (70.0\%) - Table 1.

In both groups, among men atopic dermatitis occurred more frequently $(56.6 \%$ vs. $49.2 \% ; p<0.05$ and $54.5 \%$ vs. $47.6 \% ; p<0.05$, respectively) and among women, dry skin (55.3\% vs. $47.4 \%$; $p<0.05$ and $50.0 \%$ vs. $44.5 \%$; $p<0.05$, respectively). In both groups, atopic dermatitis more frequently occurred in subjects aged below than above 18 years (59.2\% vs. 34.3\%; $p<0.01$ and $64.4 \%$ vs. $30.9 \% ; p<0.01$, respectively). While, the dry skin and skin pruritus more frequently occurred in subjects aged above 18 years (61.5\% vs. $47.3 \% ; p<0.01$; $90.2 \%$ vs. $35.7 \%$ and $52.8 \%$ vs. $33.4 \% ; p<0.01 ; 42.7 \%$ vs. $18.7 \% ; p<0.01$, respectively). In both groups, atopic dermatitis more frequently occurred in residents of rural 
Table 1. Study group characteristics

\begin{tabular}{|c|c|c|c|}
\hline Parameter & $\begin{array}{c}\text { Respondents surveyed } \\
\text { by doctors } \\
N=40.440\end{array}$ & $\begin{array}{l}\text { Respondents surveyed } \\
\text { by pharmacists } \\
N=9.035\end{array}$ & $\begin{array}{l}\text { Respondents surveyed } \\
\text { by doctors vs. surveyed } \\
\text { by pharmacists }\end{array}$ \\
\hline \multicolumn{4}{|l|}{ Gender, $n(\%)$ : } \\
\hline Female & $22485 / 55.6$ & $5583 / 61.8$ & $<0.05^{*}$ \\
\hline Male & $17955 / 44.4$ & $3451 / 38.2$ & \\
\hline \multicolumn{4}{|l|}{ Age, $n(\%)$ [years]: } \\
\hline$\leq 6$ & $16621 / 41.1$ & $2954 / 32.7$ & $<0.01^{*}$ \\
\hline $7-18$ & $10758 / 26.6$ & $1680 / 18.6$ & \\
\hline $19-25$ & $5338 / 13.2$ & $1346 / 14.9$ & \\
\hline $26-40$ & $5297 / 13.1$ & $2178 / 24.1$ & \\
\hline$>40$ & $2426 / 6.0$ & $877 / 9.7$ & \\
\hline \multicolumn{4}{|l|}{ Place of residence, $n$ (\%): } \\
\hline Rural & $12214 / 30.2$ & $2530 / 28.0$ & $<0.01^{*}$ \\
\hline Town $\leq 200000$ residents & $23050 / 57.0$ & $4210 / 46.6$ & \\
\hline City $>200000$ residents & $5176 / 12.8$ & $2295 / 25.4$ & \\
\hline \multicolumn{4}{|l|}{ Education, $n(\%)$ : } \\
\hline Primary & $5742 / 14.2$ & $1157 / 12.8$ & $<0.01^{*}$ \\
\hline Secondary & $10636 / 26.3$ & $2674 / 29.6$ & \\
\hline Higher & $6066 / 15.0$ & $1942 / 21.5$ & \\
\hline Not applicable & $17996 / 44.5$ & $3262 / 36.1$ & \\
\hline \multicolumn{4}{|l|}{ Skin symptoms, $n$ (\%): } \\
\hline Dry skin & $20945 / 51.8$ & $4328 / 47.9$ & $<0.01^{*}$ \\
\hline Skin pruritus & $15731 / 38.9$ & $6324 / 70.0$ & \\
\hline Atopic dermatitis & $21231 / 52.5$ & $4536 / 50.2$ & \\
\hline Prone to recurrent skin infections & $4772 / 11.8$ & $578 / 6.4$ & \\
\hline Other & $526 / 1.3$ & $72 / 0.8$ & \\
\hline \multicolumn{4}{|l|}{ Atoperal product use, $n(\%)$ : } \\
\hline Yes & $29400 / 72.7$ & 7201/79.7 & NS \\
\hline No & $11040 / 27.3$ & $1834 / 20.3$ & \\
\hline \multicolumn{4}{|c|}{ Type of Atoperal products use by adults, $n(\%)$ : } \\
\hline Body wash emulsion & $6126 / 46.9$ & $1069 / 24.3$ & \\
\hline Body wash & $9012 / 69.0$ & $1443 / 32.8$ & $<0.001^{*}$ \\
\hline Cream & $5642 / 43.2$ & $1162 / 26.4$ & \\
\hline Shampoo & $2834 / 21.7$ & $656 / 14.9$ & \\
\hline \multicolumn{4}{|l|}{ Type of Atoperal Baby products use [\%]: } \\
\hline Body emulsion & $16071 / 58.7$ & $1617 / 34.9$ & $<0.001^{*}$ \\
\hline Bath lotion & $14593 / 55.3$ & $1515 / 32.7$ & \\
\hline Foam wash & $3313 / 12.1$ & $533 / 11.5$ & \\
\hline Shampoo & $2300 / 8.4$ & $385 / 8.3$ & \\
\hline Cream & $9829 / 35.9$ & $630 / 13.6$ & \\
\hline
\end{tabular}


Table 1. Cont.

\begin{tabular}{|c|c|c|c|}
\hline Parameter & $\begin{array}{c}\text { Respondents surveyed } \\
\text { by doctors } \\
N=40.440\end{array}$ & $\begin{array}{c}\text { Respondents surveyed } \\
\text { by pharmacists } \\
N=9.035\end{array}$ & $\begin{array}{c}\text { Respondents surveyed } \\
\text { by doctors vs. surveyed } \\
\text { by pharmacists }\end{array}$ \\
\hline \multicolumn{4}{|c|}{ Changes observed by patients after Atoperal products use [\%]: } \\
\hline Improving of skin hydration & $22579 / 76.8$ & $5300 / 73.6$ & $<0.01^{*}$ \\
\hline Improving of skin greasiness & $14288 / 48.6$ & $3845 / 53.4$ & \\
\hline Increase in skin elasticity & $8232 / 28.0$ & $994 / 13.8$ & \\
\hline Reduction of itching & $12789 / 43.5$ & $2744 / 38.1$ & \\
\hline Acceleration of healing & $4998 / 17.0$ & $965 / 13.4$ & \\
\hline \multicolumn{4}{|c|}{ Satisfaction with Atoperal products use [\%]: } \\
\hline Unsatisfied & $59 / 0.2$ & $29 / 0.4$ & \\
\hline Moderately satisfied & $2558 / 8.7$ & $598 / 8.3$ & NS \\
\hline Satisfied & $20080 / 68.3$ & $4659 / 64.7$ & \\
\hline Very satisfied & $6703 / 22.8$ & $1915 / 26.6$ & \\
\hline \multicolumn{4}{|c|}{ The source of information about Atoperal products [\%]: } \\
\hline Doctors & $30532 / 75.5$ & $2599 / 36.1$ & $<0.001^{*}$ \\
\hline Pharmacists & 7036/17.4 & $3521 / 48.9$ & \\
\hline Friends & $1618 / 4.0$ & $425 / 5.4$ & \\
\hline Promotional handouts & $647 / 1.6$ & $295 / 4.1$ & \\
\hline Internet & $526 / 1.3$ & $346 / 4.8$ & \\
\hline Newspapers & $81 / 0.2$ & $15 / 0.2$ & \\
\hline
\end{tabular}

than urban areas (57.6\% vs. 52.7\%; $p<0.05$ and $56.8 \%$ vs. $47.1 \%$; $p<0.01$, respectively). While, the dry skin and skin pruritus more frequently occurred in urban than rural residents (58.5\% vs. $40.8 \%$; $p<0.01,54.0 \%$ vs. $35.6 \%$; $p<0.01$ and $43.1 \%$ vs. $31.1 \% ; p<0.01 ; 33.2 \%$ vs. $25.5 \%$, $p<0.01$, respectively). In the group surveyed by doctors, the dry skin and pruritus least frequency was reported by subjects with primary education, and in the group surveyed by pharmacists by subjects without education. While, the atopic dermatitis occurred most frequently among subjects with primary education and without education (data not shown).

The patients' subjective assessment of the effect of Atoperal and Atoperal Baby products on the skin symptoms

In both groups, respondents most frequently reported after Atoperal and Atoperal Baby products use improving of skin hydration andgreasiness of the skin and reduction of itching (Table 1).

In both groups, the frequency of improving of skin hydration and reduction of itching after Atoperal products use was similar among women and men $(77.9 \%$ vs. $75.5 \%$; $43.5 \%$ vs. $43.5 \%$ and $75.0 \%$ vs. $71.4 \%$; $38.9 \%$ vs. $36.8 \%$, respectively). While, in the group surveyed by doctors, the improving of greasiness of the skin was slightly more frequently reported by women than men (49.6\% vs. $47.4 \%$ ) and in the group surveyed by pharmacists, by men than women $(55.9 \%$ vs. $51.8 \%$; $p<0.05)$ - data not shown.

In the group surveyed by doctors, improving of skin hydration after Atoperal use was least frequently reported by subjects aged over 25 years (68.1\% vs. $78.8 \%$ vs. $81.6 \%$ vs. $79.5 \% ; p<0.01$ ) and in the group surveyed by pharmacists, by subjects aged 6 years and below, and $19-25$ years $(71.0 \%$ and $70.0 \%$ vs. $73.9 \%$ vs. $79.9 \%$; $p<0.05)$. Improving of the greasiness of the skin was least frequently reported by respondents aged above 40 years and $19-25$ years (35.8\% vs. $47.8 \%$ vs. $52.3 \%$ vs. $49.3 \%$ vs. $49.9 \%$; $p<0.01$ and $47.3 \%$ vs. $59.8 \%$ vs. $53.4 \%$ vs. $51.4 \%$ vs. $52.7 \%$; $p$ 0.05). In both groups, reduction of itching was least frequently reported by subjects aged 6 years and below ( $40.3 \%$ vs. $42.6 \%$ vs. $51.8 \%$ vs. $48.3 \%$ vs. $48.8 \% ; p<0.05$ and $30.5 \%$ vs. $41.5 \%$ vs. $40.5 \%$ vs. $42.8 \%$ vs. $44.8 \%$; $p<0.01$, respectively) - data not shown.

In both groups, improving of skin hydration and greasiness as well as reduction of itching was least frequently reported by rural residents $(74.3 \%$ vs. $77.5 \%$ vs. $80.5 \% ; p<0.05 ; 35.1 \%$ vs. $53.5 \%$ vs. $58.0 \% ; p<0.001$; $30.9 \%$ vs. $48.7 \%$ vs. $49.1 \% ; p<0.001$ and $67.6 \%$ vs. $75.5 \%$ vs. $77.0 \% ; p<0.05 ; 45.3 \%$ vs. $52.6 \%$ vs. $64.4 \% ; p<0.01$; $35.6 \%$ vs. $39.9 \%$ vs. $37.5 \%$; $p<0.05$, respectively) - data not shown.

In the group surveyed by doctors, improving of skin hydration was most frequently reported by respondents 
with primary education ( $84.7 \%$ vs. $74.8 \%$ vs. $69.1 \%$ vs. $78.4 \% ; p<0.01$ ) and in the group surveyed by pharmacists, subjects with higher education $(81.8 \%$ vs. $74.1 \%$ vs. $71.6 \%$ vs. $70.1 \% ; p<0.01)$. While, improving the greasiness of the skin more frequently reported subjects without education and with higher education, respectively (52.3\% vs. $50.0 \%$ vs. $44.5 \%$ vs. $44.1 \% ; p<0.05$ and $60.5 \%$ vs. $51.5 \%$ vs. $46.8 \%$ vs. $54.5 \% ; p<0.05)$. In turn, reduction of itching was more frequently reported by subjects with higher education and with primary education, respectively $(52.7 \%$ vs. $37.3 \%$ vs. $44.1 \%$ vs. $41.9 \% ; p<0.01$ and $43.3 \%$ vs. $40.1 \%$ vs. $42.8 \%$ vs. $32.4 \%$; $p<0.05$, respectively) - data not shown.

In the group surveyed by doctors, improving of skin hydration after Atoperal products use was more frequently reported by subjects with dry skin and atopic dermatitis, and in the group surveyed by pharmacists, by subjects with dry skin $(82.8 \%$ and $81.4 \%$ vs. $75.0 \%$ vs. $66.0 \%$ vs. $80.8 \% ; p<0.05$ and $85.3 \%$ vs. $66.5 \%$ vs. $77.9 \%$ vs. $65.5 \%$ vs. $57.1 ; p<0.01$, respectively). Improving of the greasiness of the skin was more frequently reported by subjects with pruritus and atopic dermatitis, and with other skin disturbances (54.8\% and $53.1 \%$ vs. $52.8 \%$ vs. $51.7 \%$ vs. $30.8 \% ; p<0.05$ and $61.9 \%$ vs. $53.0 \%$ vs. $59.6 \%$ vs. $57.1 \%$ vs. $53.8 \% ; p<0.01$, respectively). While, in both groups, reduction of itching was more frequently reported by subjects with pruritus (66.4\% vs. $47.8 \%$ vs. $43.7 \%$ vs. $56.1 \%$ vs. $34.6 \% ; p<0.001$ and $73.3 \%$ vs. $37.1 \%$ vs. $36.0 \%$ vs. $47.0 \%$ vs. $57.1 \%$; $p<0.001$, respectively) - data not shown.

\section{The satisfaction with Atoperal and Atoperal Baby products use}

In the group surveyed by doctors, one of the Atoperal products was used by $72.7 \%$ of respondents and in the group surveyed by pharmacists, by $79.7 \%$ (Table 1 ).

In both groups, over $90.0 \%$ of respondents were satisfied or very satisfied with Atoperal products use (Table 1).

The percentage of women and men unsatisfied with Atoperal products use was similar in both study groups (Table 2). While, in both groups, men were slightly more often satisfied than women with Atoperal products use and women slightly more often were very satisfied (Table 2).

In the group surveyed by doctors, a higher percentage of patients unsatisfied with Atoperal products use was observed among respondents aged 19-25 years and 26-40 years and very satisfied among respondents aged over 40 years, while, in the group surveyed by pharmacists, among respondents aged over 40 years. However, in both groups, the percentage of subjects very satisfied was the highest among respondents aged over 40 years (Table 2).

In the group surveyed by doctors, residents of big cities were least frequently unsatisfied with Atoperal products use (Table 2 ). In addition, this subgroup was more frequently very satisfied with Atoperal products use (Table 2). While, in the group surveyed by pharmacists, residents of big cities were most frequently unsatisfied with Atoperal products use and residents of smaller towns were most frequently very satisfied with Atoperal products use (Table 2 ).

In both study groups, subjects with higher education were most frequently unsatisfied and very satisfied with Atoperal products use (Table 2).

In a group surveyed by doctors, respondents with pruritus and atopic dermatitis were most frequently unsatisfied with Atoperal products use, and in a group surveyed by pharmacists, these were respondents with other skin disturbances (Table 2). While, in both groups, respondents prone to recurrent skin infections were most frequently very satisfied with Atoperal products use (Table 2).

\section{Sources of information about Atoperal products}

In the group surveyed by doctors, $75.5 \%$ of respondents obtained the information about Atoperal products from doctors and $17.4 \%$ from pharmacists. While, in the group surveyed by pharmacists, $48.9 \%$ obtained such information from pharmacists and $36.1 \%$ from doctors. Promotional handouts, Internet and newspapers were the marginal sources of obtaining information by respondents involved in this study (Table 1).

In the group surveyed by doctors, women slightly more than men obtained information about Atoperal products from doctors (76.5\% vs. $74.4 \%$ ) and men from pharmacists (16.7\% vs. 18.2\%). While, in a group surveyed by pharmacists, women more frequently than men obtained information about Atoperal products from pharmacists (51.6\% vs. $44.2 \% ; p<0.01)$ and men from doctors (41.8\% vs. $32.5 \% ; p<0.01)$ - Figure 1 .

In the group surveyed by doctors, independent of age subgroups, over $70.0 \%$ of respondents obtained information about Atoperal products from doctors, slightly more frequently they were guardians of children aged 6 years and below (79.6\% vs. $73.3 \%$ vs. $71.0 \%$ vs. $70.5 \%$ vs. $76.4 \%$; $p<0.05)$. While, in the group surveyed by pharmacists, guardians of subjects aged 18 years and below received information about Atoperal products least frequently from pharmacists (46.1\% and $46 . \%$ vs. $48.6 \%$ vs. $55.4 \%$ vs. $47.5 \%$; $p<0.05)$. In both groups, respondents aged over 18 years themselves obtained most frequently information from promotional handouts, Internet and newspapers, and friend. However, this frequency was higher in a group surveyed by pharmacists than by doctors (data not shown).

In both study groups, the information about Atoperal products was obtained from doctors the least frequently by residents of big cities. In the group surveyed by doctors, pharmacists were the least frequent source of information about Atoperal products for residents of smaller towns and in the group surveyed by pharmacists, for rural residents (Figure 2). 


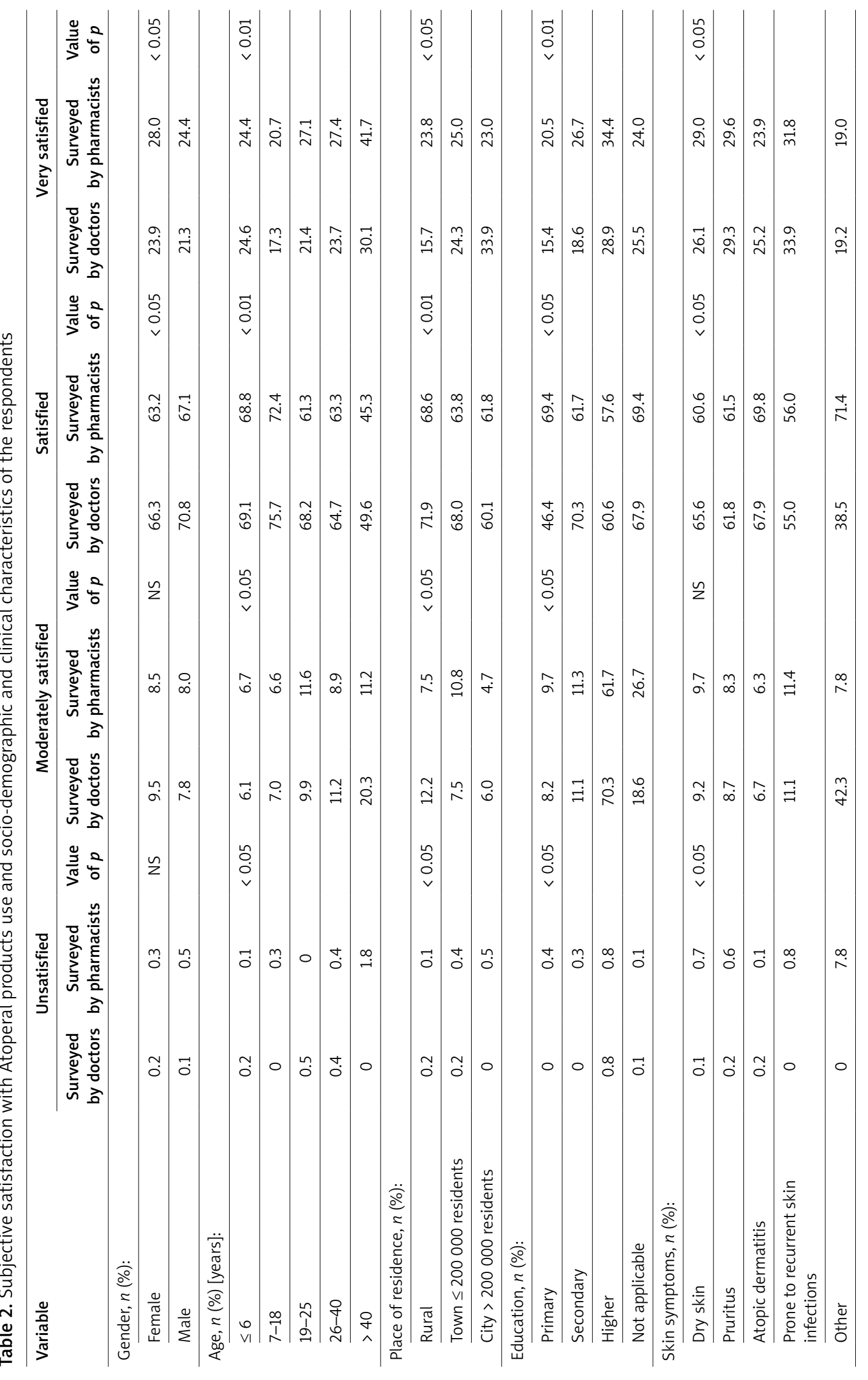




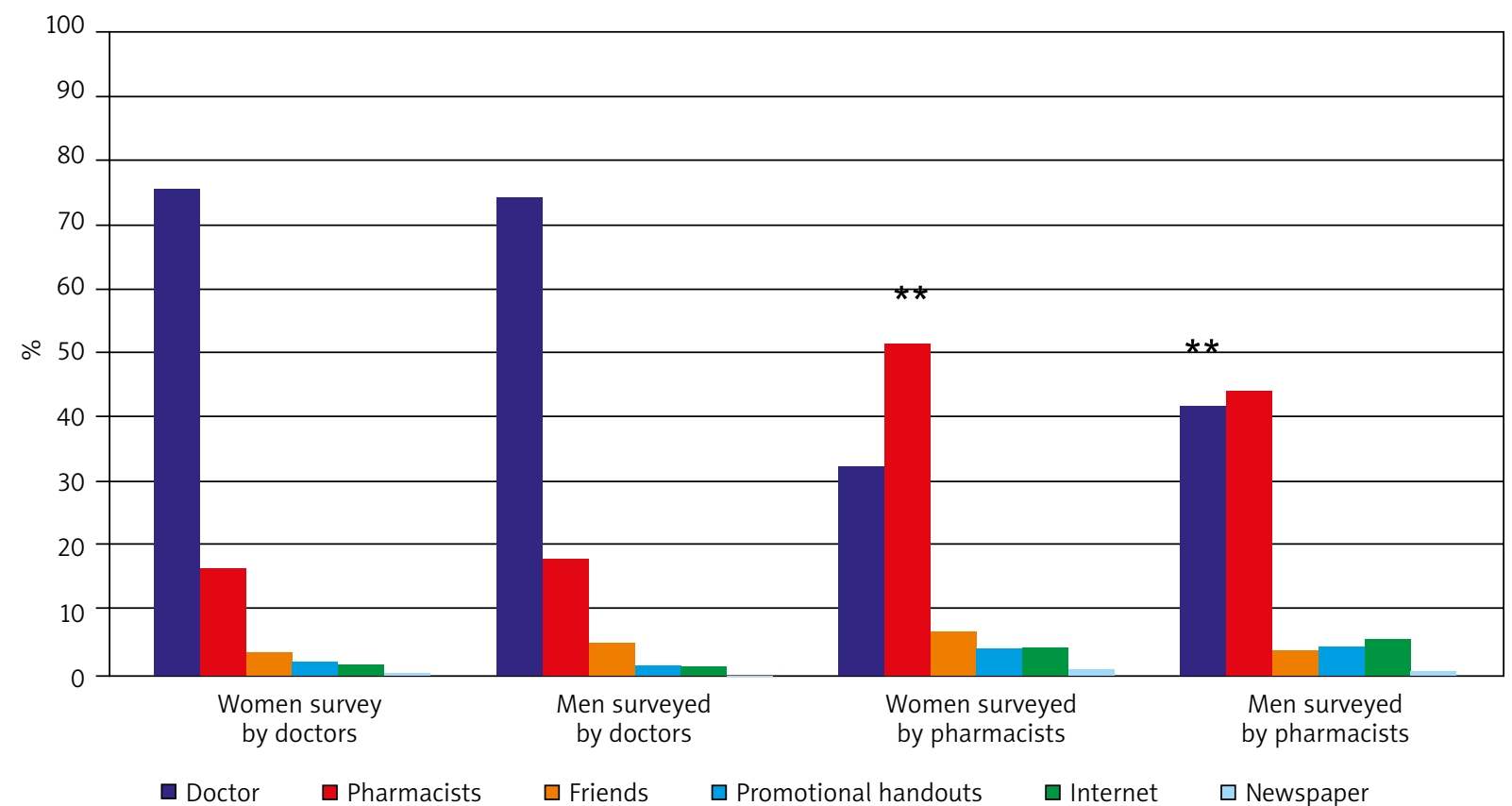

${ }^{* *} p<0.01$ women vs. men.

Figure 1. Sources of information about Atoperal products according to gender

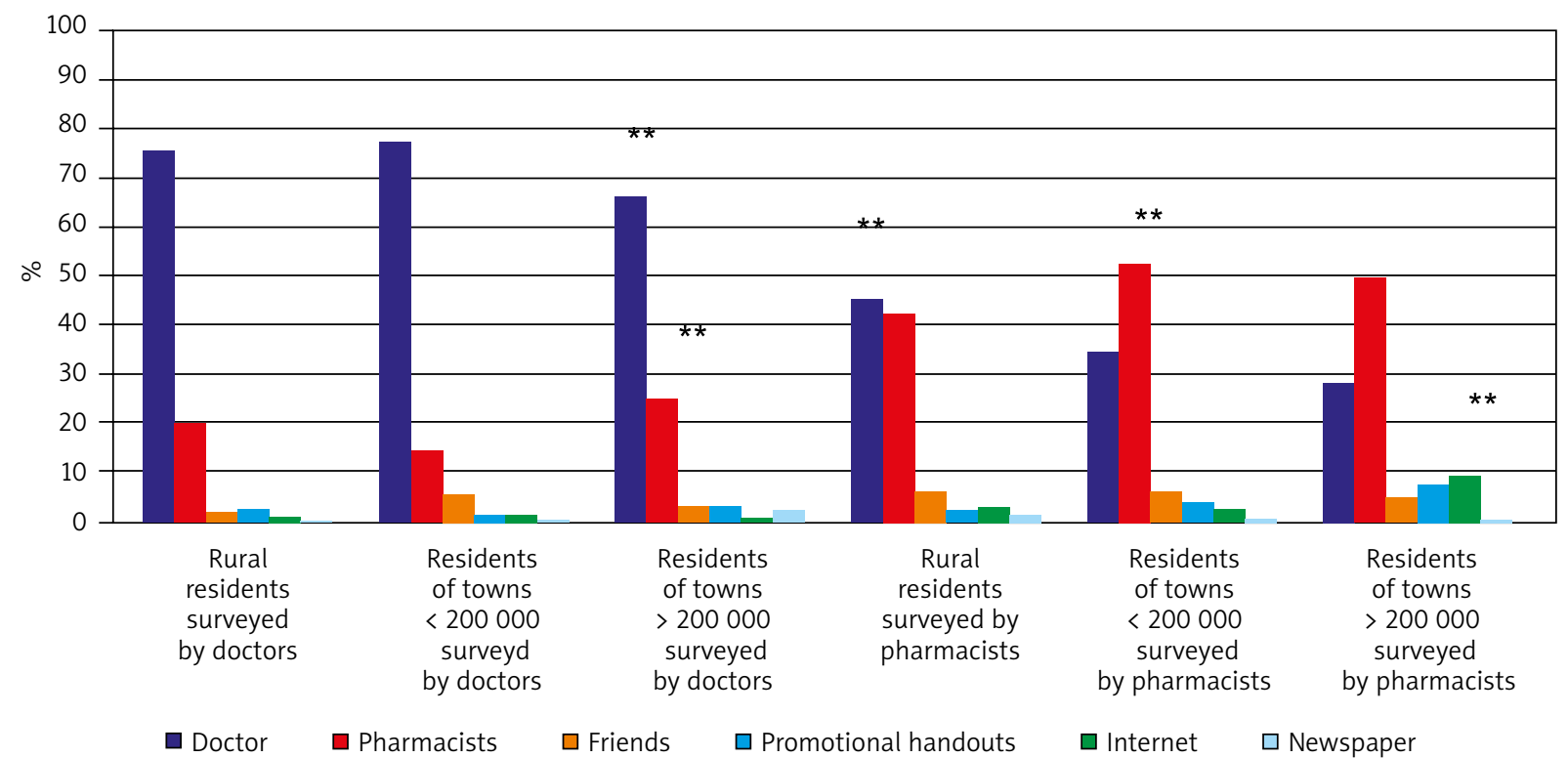

${ }^{* *} p<0.01$ surveyed by doctors vs. surveyed by pharmacists.

Figure 2. Sources of information about Atoperal products according to the place of residence

In both groups, subjects with higher education obtained the information about Atoperal products from doctors least frequently. While, in a group surveyed by doctors, subjects with higher education obtained the information about Atoperal products from pharmacists least frequently and in a group surveyed by pharmacists, subjects with primary education. In addition, in both groups, Internet was the most frequent source of infor- mation about Atoperal products for subjects with higher education (Figure 3).

In a group surveyed by doctors, doctors more frequently than pharmacists were the source of information about Atoperal products for subjects with atopic dermatitis and prone to recurrent dermatitis (78.4\% vs. 45.0\%; $p<0.001$ and $78.3 \%$ vs. $39.4 \%$; $p<0.001$, respectively). While, in agroup surveyed by pharmacists, pharmacists 


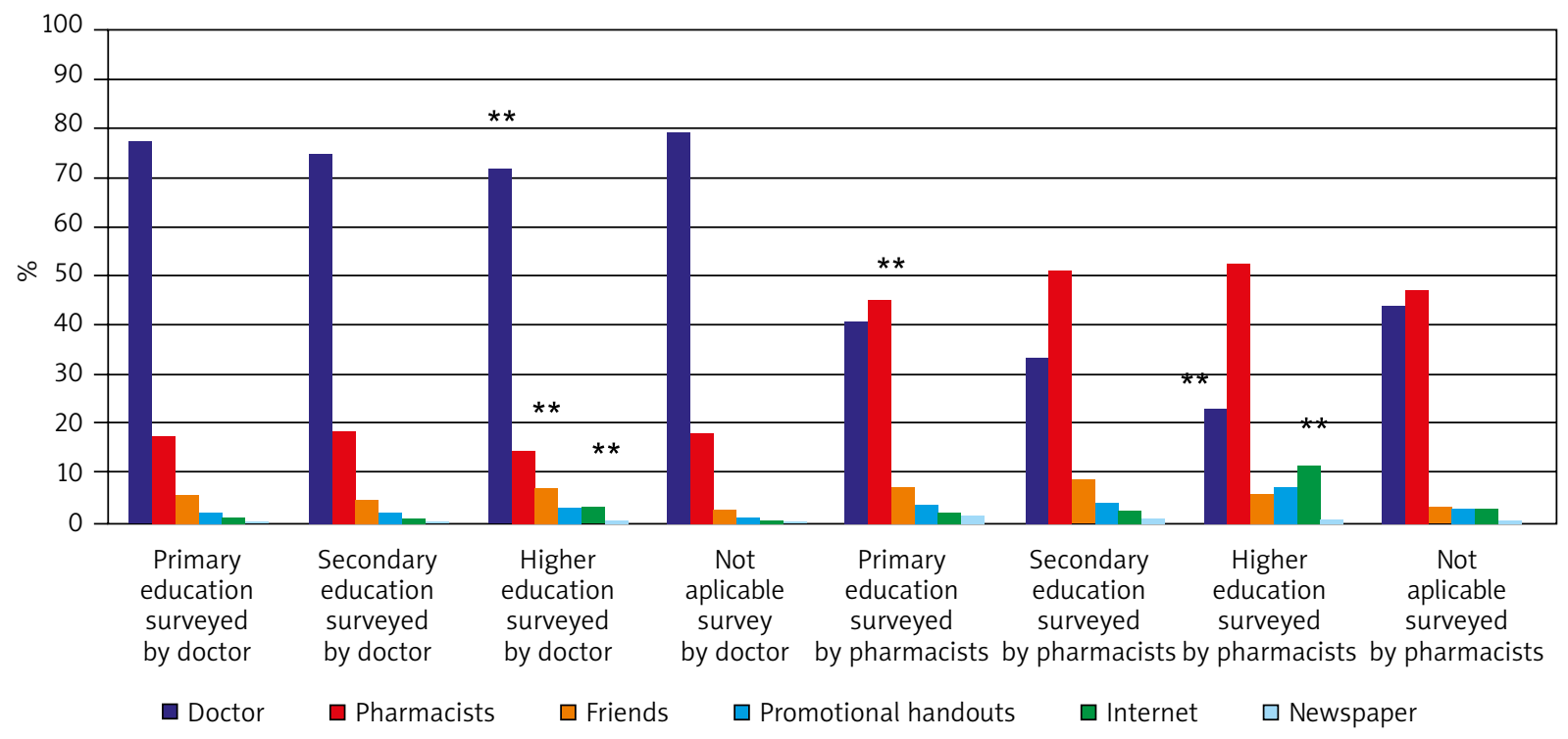

${ }^{* *} p<0.01$.

Figure 3. Sources of information about Atoperal products according to the education level

more frequently than doctors were the source of information ( $42.3 \%$ vs. $13.8 \%$ and $39.4 \%$ vs. $12.8 \%$, respectively) - data not shown.

\section{Discussion}

The presented study assessed the effect of satisfaction with one of brands of dermocosmetics use is the first large survey performed in the Polish population.

The most frequent skin problem in a group surveyed by doctors was atopic dermatitis, and in a group surveyed by pharmacists, skin itching. In both groups, dry skin occurred slightly more frequently among women and atopic dermatitis among men. It is in contrast to the previously published epidemiological study conducted among residents of the Lodz region, in which there was a similar frequency of atopic dermatitis among adult women and men [4]. While, in the Polish study conducted in children similar to the present study, atopic dermatitis occurred more frequently among boys than girls [5]. In addition, a study performed in the German population revealed the association between allergic dermatitis and male gender [6]. Considering that most of the study population were persons below 18 years of age, the results are consistent with previous observations showing the relationship between atopic dermatitis and male gender. This is in accordance with the results of other studies that have shown a higher frequency of atopic dermatitis among the population below 18 years than in adults [1] and the present study that has also shown a higher prevalence of atopic dermatitis in children and adolescents than in adults, regardless of the place of the survey. However, because the questionnaire included only age groups of 6 years and below and 7-18 years and not the age of the respondents, it is impossible to clearly show whether post-puberty is followed by a significant reduction in the percentage of subjects with atopic dermatitis. On the other hand, it seems that about the self-restraint this disease with age indicates a higher incidence of dryness and itching of the skin in the both groups of adults respondents. However, it is interesting that frequency of people prone to recurrent skin infections increased with age regardless of the place of the survey.

Contrary to the previously published study [4] that shown an increased risk of atopic dermatitis among adults living in the city center, atopic dermatitis occurred least frequently among big city residents. However, the analysis did not take into account the age or city and rural residence. On the other hand, in the children population in other studies [5, 7], there was no association between the occurrence of atopic dermatitis and the place of residence. In addition, the results of a study conducted in the Finish population showed no differences in the incidence of allergic diseases among those living in rural areas, but was raised in non-agricultural families and city residents [8]. It should be noted that this aspect was not analyzed in this study.

One of the described risk factors for atopic dermatitis was a lower socioeconomic status including a low education level [9]. However, a frequent occurrence of atopic dermatitis among subjects without education in a group surveyed by doctors and among subjects with primary education in a group surveyed by pharmacists is not a confirmation of these results and the effect of the age structure of respondents. It should be noted that obtained results suggested that parents of younger children more often look for help from doctors. 
As mentioned above, the important part of treatment of atopic dermatitis and skin disturbances that do not meet the criteria for this diagnosis is dermocosmetics containing emollients use [3]. On the Polish market there are several brands of these dermocosmetics and in the present study the Atoperal brand was assessed. In both study groups, respondents most frequently reported improving of skin hydration and greasiness of the skin and reduction of itching after Atoperal products use. The percentage of women and men who reported improving of skin hydration and reduction of itching was similar in both study groups. It should be also noted that these effects in both groups were most frequently reported among children aged 6 years and below. The interesting observation, but difficult to explain, is the least frequency of rural residents reporting these effects. It is in contrast to the results of a previously published study that has shown greater severity of skin disturbances in urban residents [4]. In the present study, the subjective effects reported by respondents were analyzed and these may depend on expectations. Since the questionnaire used in this study did not include these factors, further studies are necessary to explained this association. However, this hypothesis may be partially supported by the observations that residents of big cities were more frequently very satisfied with Atoperal products use. The benefits of using Atoperal products are shown by an observation that in both groups, improving of skin hydration was reported more frequently by subjects with dry skin and in a group surveyed by doctors, also subjects with atopic dermatitis. In addition, the effectiveness of Atoperal products in pruritus relieving is supported by the fact that subjects with pruritus more frequently reported reduction of itching.

The good quality and effectiveness of Atoperal dermocosmetics confirms satisfaction of respondents with their use. In both study groups, over $90.0 \%$ of respondents were satisfied and very satisfied with Atoperal products use. In addition, this is supported by the results shown that doctors and pharmacists were the main source of the information about Atoperal products for respondents in this study. On the other hand, the present study has shown that subjects with skin disturbances rarely actively look for information about dermocosmetics. Adults with higher education frequently actively look for such information.

The study has several limitations. The most important one is the lack of the control group using dermocosmetics of other brands. The second limitation is the self-reported assessment of effectiveness.

However, the strength of the study is the large study group which is representative of the Polish population and that this study is a multicenter one.

\section{Conclusions}

Atopic dermatitis was diagnosed most frequently in a group surveyed by doctors and its prevalence was higher among men, subjects below 18 years and rural residents. The main skin disturbance that occurred in a group surveyed by pharmacists was skin pruritus, frequently among subjects above 18 years and urban residents. The main effect of Atoperal products use independent of the place of the survey was improving of skin hydration and greasiness of the skin and reduction of itching. In the study population, there was a high level of satisfaction with the use of Atoperal products. Doctors and pharmacists were the main sources of information about Atoperal products depending on the place of the survey. The respondents rarely found information about these products themselves, mainly in the Internet.

\section{Conflict of interest}

The author declares no conflict of interest.

\section{References}

1. Eichenfield LF, Fowler JF Jr, Rigel DS, Taylor SC. Natural advances in eczema care. Cutis 2007; 80 (6 Suppl): 2-16.

2. Samochocki Z, Dejewska J. A comparison of criteria for diagnosis of atopic dermatitis in children. World J Pediatr 2012; 8: 355-8.

3. Szepietowski J, Kaszuba A, Adamski Z, Placek W, Salomon J. Emollients in dermatological treatment: position paper of the experts' group. Dermatol Klin 2011; 13: 209-14.

4. Kupryś-Lipińska I, Elgalal A, Kuna P. Epidemiology of atopic dermatitis in general population of Lodz province's citizens. Pneumonol Alergol Pol 2009; 77: 145-51.

5. Stelmach W, Korzeniewska A, Krakowiak J, et al. Retrospective analysis of the results of Allergic Diseases Prevention Program in children in the Lódź area. Pneumonol Alergol Pol 2004; 72: 192-7.

6. Schafer T, Vieluf D, Behrendt H, et al. Atopic eczema and other manifestations of atopy: results of the study in East and West Germany. Allergy 1996; 51: 532-9.

7. Taylor MR, Holland CV, O'Lorcain P. Eczema and hay fever in schoolchildren. Ir Med J 1996; 89: 229-30.

8. Kilpelainen M, Terho EO, Helenius H, Koskenvuo M. Farm environment in childhood prevents the development of allergies. Clin Exp Allergy 2000; 30: 201-8.

9. Ring J, Kramer U, Schafer T, et al. Environmental risk factors for respiratory and skin atopy: results from epidemiological studies informer East and West Germany. Int Arch Allergy Immunol 1999; 118: 403-7. 